\title{
Purdue University Center for Cancer Research
}

National Cancer Institute

\section{Source}

National Cancer Institute. Purdue University Center for Cancer Research. NCI Thesaurus. Code $C 39502$.

The mission of the Purdue University Center for Cancer Research is to promote discovery in the areas of biological phenomena, new chemical entities, and new technology that leads to the development of innovative instrumentation, new diagnostic tools, and novel therapeutics. The Center's goal is to find ways to detect cancer sooner and treat it more effectively. It was designated as an NCl cancer center in 1978. 